\title{
IMA Commission on New Minerals, Nomenclature and Classification (CNMNC) NEWSLETTER 47
}

\section{New minerals and nomenclature modifications approved in 2018 and 2019}

Ritsuro Miyawaki (Chairman, CNMNC - incoming) ${ }^{1}$, Ulf Hålenius (Chairman, CNMNC - outgoing)², Frédéric Hatert (ViceChairman, CNMNC) ${ }^{3}$, Marco Pasero (Vice-Chairman, CNMNC) ${ }^{4 *}$ and Stuart J. Mills (Secretary, CNMNC) ${ }^{5}$

${ }^{1}$ Department of Geology and Paleontology, National Museum of Nature and Science, 4-1-1 Amakubo, Tsukuba 305-0005, Japan - miyawaki@kahaku.go.jp;

${ }^{2}$ Department of Geosciences, Naturhistoriska Riksmuseet, Box 50007, SE-104 05 Stockholm, Sweden - ulf.halenius@nrm.se; ${ }^{3}$ Laboratoire de Minéralogie, Université de Liège, B-4000 Liège, Belgium - fhatert@ulg.ac.be; ${ }^{4}$ Dipartimento di Scienze della Terra, Università di Pisa, Via Santa Maria 53, I-56126 Pisa, Italy - marco.pasero@ unipi.it; and ${ }^{5}$ Geosciences, Museum Victoria, PO Box 666, Melbourne, Victoria 3001, Australia - smills@museum.vic.gov.au

The information given here is provided by the IMA Commission on New Minerals, Nomenclature and Classification for comparative purposes and as a service to mineralogists working on new species.

Each mineral is described in the following format:

Mineral name, if the authors agree on its release prior to the full description appearing in press

Chemical formula

Type locality

Full authorship of proposal

E-mail address of corresponding author

Relationship to other minerals

Crystal system, Space group; Structure determined, yes or no

Unit-cell parameters

Strongest lines in the X-ray powder diffraction pattern

Type specimen repository and specimen number

Citation details for the mineral prior to publication of full description

Citation details concern the fact that this information will be published in the Mineralogical Magazine on a routine basis, as well as being added month by month to the Commission's web site.

It is still a requirement for the authors to publish a full description of the new mineral.

\section{NO OTHER INFORMATION WILL BE RELEASED BY THE COMMISSION}

\section{NEW MINERAL PROPOSALS APPROVED IN DECEMBER 2018}

IMA No. 2018-109

Avdeevite

$(\mathrm{Na}, \mathrm{Cs})\left(\mathrm{Be}_{2} \mathrm{Li}\right) \mathrm{Al}_{2}\left(\mathrm{Si}_{6} \mathrm{O}_{18}\right)$

Khat Chey (Kat Chay) mine, near the Khetchel village, Molo quarter, Momeik township, Kyaukme district, Shan State, Myanmar (2318'42”N , 9651'54”E)

Atali A. Agakhanov*, Dmitry A. Stepanenko, Natalia V. Zubkova, Igor V. Pekov, Leonid A. Pautov, Anatoly V. Kasatkin, Vladimir Y. Karpenko, Vitaliya A. Agakhanova, Radek Škoda and Sergey N. Britvin

*E-mail: atali99@mail.ru

Beryl group
Hexagonal: $P 6 / m m c$; structure determined $a=9.2287(4), c=9.2610(3) \AA$

7.96(82), 4.60(31), 3.99(20), 3.26(100), 3.02(37), 2.866(84), 2.524(13), 1.742(19)

Type material is deposited in the collections of the Fersman Mineralogical Museum, Russian Academy of Sciences, Leninskiy Prospekt 18-2, Moscow 119071, Russia, registration number 5279/1 How to cite: Agakhanov, A.A., Stepanenko, D.A., Zubkova, N.V., Pekov, I.V., Pautov, L.A., Kasatkin, A.V., Karpenko, V.Y., Agakhanova, V.A., Škoda, R. and Britvin, S.N. (2019) Avdeevite, IMA 2018-109. CNMNC Newsletter No. 47, February 2019, page 143; Mineralogical Magazine, 83, 143-147.

IMA No. 2018-110

Nickeltyrrellite

$\mathrm{CuNi}_{2} \mathrm{Se}_{4}$

El Dragón mine, $30 \mathrm{~km}$ SW of Potosí, Quijarro province, Bolivia (19²9’23.90”S, 6555’00.60”W, $4160 \mathrm{~m}$ a.s.l.) 
Hans-Jürgen Förster*, Chi Ma, Günter Grundmann, Luca Bindi and Christopher J. Stanley

${ }^{\star}$ E-mail: forhj@gfz-potsdam.de

Spinel supergroup

Cubic: $F d \overline{3} m$

$a=9.99 \AA$

X-ray powder diffraction pattern not available

Type material is deposited in the mineralogical collections of the Natural History Museum, London, catalogue number BM2018,19 (holotype), and the Mineralogical State Collection Munich - Museum Reich der Kristalle, inventory number MSM 73587 (cotype)

How to cite: Förster, H.-J., Ma, C., Grundmann, G., Bindi, L. and Stanley C.J. (2019) Nickeltyrrellite, IMA 2018-110. CNMNC Newsletter No. 47, February 2019, page 143; Mineralogical Magazine, 83, 143-147.

\section{IMA No. 2018-114}

Pseudomarkeyite

$\mathrm{Ca}_{8}\left(\mathrm{UO}_{2}\right)_{4}\left(\mathrm{CO}_{3}\right)_{12} \cdot 21 \mathrm{H}_{2} \mathrm{O}$

Markey mine, Red Canyon, White Canyon District, San Juan Co., Utah, USA $\left(37^{\circ} 32^{\prime} 57^{\prime \prime} \mathrm{N}, 110^{\circ} 18^{\prime} 08^{\prime \prime} \mathrm{W}\right)$

Anthony R. Kampf*, Travis A. Olds, Jakub Plášil, Peter C. Burns and Joe Marty

*E-mail: akampf@nhm.org

Closely related to markeyite

Monoclinic: $P 2{ }_{1} / m$; structure determined

$a=17.531(3), b=18.555(3), c=9.130(3) \AA, \beta=103.95(3)^{\circ}$

8.73(86), 6.28(100), 5.70(72), 4.65(80), 4.293(55), 4.082(47),

3.973(25), 2.811(59)

Type material is deposited in the mineralogical collections of the Natural History Museum of Los Angeles County, 900 Exposition Boulevard, Los Angeles, CA 90007, USA, catalogue number 67091

How to cite: Kampf, A.R., Olds, T.A., Plášil, J., Burns, P.C. and Marty, J. (2019) Pseudomarkeyite, IMA 2018-114. CNMNC Newsletter No. 47, February 2019, page 144; Mineralogical Magazine, 83, 143-147.

\section{IMA No. 2018-115}

Magnesiobermanite

$\mathrm{MgMn}_{2}^{3+}\left(\mathrm{PO}_{4}\right)_{2}(\mathrm{OH})_{2} \cdot 4 \mathrm{H}_{2} \mathrm{O}$

White Rock No. 2 quarry, Bimbowrie Conservation Park, $24 \mathrm{~km} \mathrm{~N}$ of Olary, South Australia, Australia (32 $4^{\prime} 51^{\prime \prime} \mathrm{S}$, $\left.140^{\circ} 19^{\prime} 45^{\prime \prime} \mathrm{E}\right)$

Peter Elliott*

${ }^{\star}$ E-mail: peter.elliott@adelaide.edu.au

The $\mathrm{Mg}$ analogue of bermanite

Monoclinic: $P 2_{1}$; structure determined

$a=5.421(1), b=19.072(4), c=5.389(1) \AA, \beta=110.21(3)^{\circ}$

9.533(100), 5.089(8), 4.878(3), 4.772(21), 3.634(3), 3.246(3), $3.046(2), 2.892(6)$

Type material is deposited in the mineralogical collections of the South Australian Museum, North Terrace, Adelaide, South Australia, Australia, registration number G34762

How to cite: Elliott, P. (2019) Magnesiobermanite, IMA 2018-115. CNMNC Newsletter No. 47, February 2019, page 144; Mineralogical Magazine, 83, 143-147.
IMA No. 2018-116

Perbøeite-(La)

$\left(\mathrm{CaLa}_{3}\right)\left(\mathrm{Al}_{3} \mathrm{Fe}^{2+}\right)\left[\mathrm{Si}_{2} \mathrm{O}_{7}\right]\left[\mathrm{SiO}_{4}\right]_{3} \mathrm{O}(\mathrm{OH})_{2}$

Mochalin Log REE deposit, $14 \mathrm{~km} \mathrm{~N}$ of the city of Kyshtym,

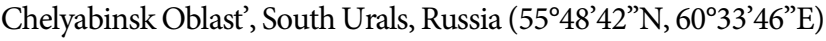
Anatoly V. Kasatkin*, Igor V. Pekov, Natalia V. Zubkova, Nikita V. Chukanov, Radek Škoda, Yury S. Polekhovsky, Dmitriy I. Belakovskiy, Atali A. Agakhanov, Aleksey M. Kuznetsov and Dmitry Y. Pushcharovsky

*E-mail: anatoly.kasatkin@gmail.com

Gatelite supergroup

Monoclinic: $P 2_{1} / m$; structure determined

$a=8.9652(4), b=5.7306(2), c=17.6770(9) \AA, \beta=116.053(6)^{\circ}$

15.85(83), $\quad 8.03(41), \quad 4.665(68), \quad 3.523(99), \quad 3.116(56)$, 3.009(100), 2.874(55), 2.640(54)

Type material is deposited in the collections of the Fersman Mineralogical Museum, Russian Academy of Sciences, Leninskiy Prospekt 18-2, Moscow 119071, Russia, registration number 5253/1

How to cite: Kasatkin, A.V., Pekov, I.V., Zubkova, N.V., Chukanov, N.V., Škoda, R., Polekhovsky, Y.S., Belakovskiy, D.I., Agakhanov, A.A., Kuznetsov, A.M. and Pushcharovsky, D.Y. (2019) Perbøeite-(La), IMA 2018-116. CNMNC Newsletter No. 47, February 2019, page 144; Mineralogical Magazine, 83, 143-147.

IMA No. 2018-043

Nikmelnikovite

$\mathrm{Ca}_{12} \mathrm{Fe}^{2+} \mathrm{Fe}_{3}^{3+} \mathrm{Al}_{3}\left(\mathrm{SiO}_{4}\right)_{6}(\mathrm{OH})_{20}$

Kovdor massif, Kola peninsula, Murmansk Region, Russia $\left(67^{\circ}\right.$ $35^{\prime} 21^{\prime \prime} \mathrm{N}, 30^{\circ} 27^{\prime} 33^{\prime \prime} \mathrm{E}$ )

Sergey V. Krivovichev*, Victor N. Yakovenchuk, Taras L. Panikorovskii, Eugeny E. Savchenko, Yakov A. Pakhomovsky, Julia A. Mikhailova, Ekaterina A. Selivanova, Galijabanu I. Kadyrova and Gregory Y. Ivanyuk

^E-mail: skrivovi@mail.ru

Garnet supergroup

Trigonal: $R \overline{3}$; structure determined $a=17.2072(6), c=10.5689(4) \AA$

8.57(65), 3.042(58), 2.720(100), 2.483(27), 2.223(28), 1.975(30), $1.687(31), 1.626(32)$

Type material is deposited in the collections of the Mineralogical Museum, St. Petersburg State University, Russia, catalogue No. 1/19691

How to cite: Krivovichev, S.V., Yakovenchuk, V.N., Panikorovskii, T.L., Savchenko, E.E., Pakhomovsky, Y.A., Mikhailova, J.A., Selivanova, E.A., Kadyrova, G.I. and Ivanyuk, G.Y. (2019) Nikmelnikovite, IMA 2018-043. CNMNC Newsletter No. 47, February 2019, page 144; Mineralogical Magazine, 83, 143-147.

IMA No. 2018-103

Carmeltazite

$\mathrm{ZrAl}_{2} \mathrm{Ti}_{4} \mathrm{O}_{11}$

In pockets of trapped melt interstitial to, or included in, corundum crystals, Mt. Carmel volcanics, Israel

William L. Griffin, Sarah E.M. Gain, Luca Bindi*, Verez Toledo, Fernando Cámara, Martin Saunders and Suzanne Y. O’Reilly

^E-mail: luca.bindi@unifi.it 
New structure type

Orthorhombic: Pnma; structure determined

$a=14.0951(9), b=5.8123(4), c=10.0848(7) \AA$

5.04(65), 4.09(60), 2.961(100), 2.885(40), 2.732(30), 2.051(25),

$2.047(60), 1.456(30)$

Type material is deposited in the mineralogical collections of the Museo di Storia Naturale, Università di Firenze, Via La Pira 4, I-50121, Firenze, Italy, catalogue number 3293/I

How to cite: Griffin, W.L., Gain, S.E.M., Bindi, L., Toledo, V., Cámara, F., Saunders, M. and O’Reilly, S.Y. (2019) Carmeltazite, IMA 2018-103. CNMNC Newsletter No. 47, February 2019, page 144; Mineralogical Magazine, 83, 143-147.

\section{NEW MINERAL PROPOSALS APPROVED IN JANUARY 2019}

\section{IMA No. 2018-111}

Llantenesite

$\mathrm{Cu}_{6} \mathrm{Al}\left[\mathrm{SeO}_{4}\right](\mathrm{OH})_{12} \mathrm{Cl} \cdot 3 \mathrm{H}_{2} \mathrm{O}$

Mina San Pedro, Cuesta de Los Llantenes mining district, NW Jagüé, Vinchina Department, La Rioja Province, Argentina (2820-40’S, 68³0-45’W)

Christian L. Lengauer ${ }^{\star}$, Martin Ende, Dan Topa, Raúl Lira and Werner H. Paar

*E-mail: christian.lengauer@univie.ac.at

The Se analogue of spangolite

Trigonal: $P 31 c$; structure determined

$a=8.2603(3), c=14.5953(4) \AA$

7.297(100), 3.650(40), 3.254(5), 2.535(6), 2.363(4), 1.984(2), $1.824(3), 1.552(2)$

Type material is deposited in the mineralogical collections of the Naturhistorisches Museum Wien, Burgring 7, 1010 Wien, Austria, catalogue No. O574

How to cite: Lengauer, C.L., Ende, M., Topa, D., Lira, R. and Paar, W.H. (2019) Llantenesite, IMA 2018-111. CNMNC Newsletter No. 47, February 2019, page 145; Mineralogical Magazine, 83, 143-147.

\section{IMA No. 2018-113}

Donwilhelmsite

$\mathrm{CaAl}_{4} \mathrm{Si}_{2} \mathrm{O}_{11}$

In the lunar meteorite Oued Awlitis 001, found on January 15, 2014 in the Boujdour Province, Laâyoune-Sakia El Hamra Region, Western Sahara $\left(25.954^{\circ} \mathrm{N}, 12.493^{\circ} \mathrm{W}\right)$

Jörg Fritz ${ }^{*}$, Ansgar Greshake, Mariana Klementova, Richard Wirth, Lukas Palatinus, Vera Assis Fernandes, Ute Böttger and Ludovic Ferrière

*E-mail: joerg.fritz@kino-heppenheim.de

Structurally related to zagamiite

Hexagonal: $P 6_{3} / m m c$; structure determined

$a=5.44(1), c=12.76(3) \AA$

$\mathrm{X}$-ray powder diffraction pattern not available

Type material is deposited in the mineralogical collections of the Naturhistorisches Museum Wien, Burgring 7, 1010 Wien, Austria, catalogue no. NHMW-O104

How to cite: Fritz, J., Greshake, A., Klementova, M., Wirth, R., Palatinus, L., Assis Fernandes, V., Böttger, U. and Ferrière, L. (2019) Donwilhelmsite, IMA 2018-113. CNMNC Newsletter No. 47, February 2019, page 145; Mineralogical Magazine, 83, 143-147.
IMA No. 2018-119

Glikinite

$\mathrm{Zn}_{3} \mathrm{O}\left(\mathrm{SO}_{4}\right)_{2}$

Arsenatnaya fumarole, Second scoria cone of the Northern Breakthrough of the Great Tolbachik Fissure Eruption, Tolbachik volcano, Kamchatka peninsula, Far-Eastern Region, Russia $\left(55^{\circ} 41^{\prime} \mathrm{N}, 160^{\circ} 14^{\prime} \mathrm{E}, 1200 \mathrm{~m}\right.$ asl)

Evgeniy V. Nazarchuk, Oleg I. Siidra*, Diana O. Nekrasova, Artem S. Borisov and Vladimir V. Shilovskikh

${ }^{\star}$ E-mail: o.siidra@spbu.ru

Known synthetic analogue

Monoclinic: $P 2_{1} / m$; structure determined

$a=7.30(2), b=6.59(1), c=7.84(1) \AA, \beta=117.14(3)^{\circ}$

$6.968(56), \quad 3.942(52), \quad 3.483(100), \quad 3.294(49), \quad 2.936(43)$, 2.533(63), 2.507(63), 2.394(86)

Type material is deposited in the collections of the Mineralogical Museum, Department of Mineralogy, St Petersburg State University, St Petersburg, Russia, specimen number 1/19695

How to cite: Nazarchuk, E.V., Siidra, O.I., Nekrasova, D.O., Borisov, A.S. and Shilovskikh, V.V. (2019) Glikinite, IMA 2018-119. CNMNC Newsletter No. 47, February 2019, page 145; Mineralogical Magazine, 83, 143-147.

IMA No. 2018-120

Šlikite

$\mathrm{Zn}_{2} \mathrm{Mg}\left(\mathrm{CO}_{3}\right)_{2}(\mathrm{OH})_{2} \cdot 4 \mathrm{H}_{2} \mathrm{O}$

Vladimír mine (II. level), Plavno, Jáchymov ore district, Western Bohemia, Czech Republic

Jiří Sejkora*, Ian E. Grey, Anthony R. Kampf, W. Gus Mumme, Bohuslav Bureš and Jiř́ Čejka

*E-mail: jiri_sejkora@nm.cz

New structure type

Triclinic: $P \overline{1}$; structure determined

$a=6.335(4), b=6.340(1), c=13.923(4) \AA$, $\alpha=99.985(7), \beta=$ 92.74(1), $\gamma=114.93(2)^{\circ}$

13.57(100), 4.525(31), 3.573(2), 3.406(5), 3.394(5), 3.326(1), 2.996(3), 2.773(3)

Type material is deposited in the mineralogical collection of the National Museum, Prague, Czech Republic, catalogue number P1P 46/2018, and the Natural History Museum of Los Angeles County, 900 Exposition Boulevard, Los Angeles, CA 90007, USA, catalogue number 67057

How to cite: Sejkora, J., Grey, I.E., Kampf, A.R., Mumme, W.G., Bureš, B. and Čejka, J. (2019) Šlikite, IMA 2018-120. CNMNC Newsletter No. 47, February 2019, page 145; Mineralogical Magazine, 83, 143-147.

IMA No. 2018-124

Orthocuproplatinum

$\mathrm{Pt}_{3} \mathrm{Cu}$

Lubero region, Democratic Republic of the Congo

Alexandre Raphael Cabral*, Roman Skála, Anna Vymazalová, Jaroslav Maixner, Chris J. Stanley, Bernd Lehmann and Jacques Jedwab

*E-mail: arcab@ufmg.br

Known synthetic analogue

Orthorhombic: $\mathrm{Cmmm}$; structure determined

$a=7.681(1), b=5.4318(8), c=2.7502(4) \AA$

2.337(11), 2.236(100), 2.217(97), 1.932(61), 1.920(30), 1.362(36),

$1.169(24), 1.161(23)$ 
Type material is deposited in the mineralogical collection of "Geosammlung", Technische Universität Clausthal, Adolph-Roemer-Strasse 2A, D-38678 Clausthal-Zellerfeld, Germany, catalogue number 11331

How to cite: Cabral, A.R., Skála, R., Vymazalová, A., Maixner, J., Stanley, C.J., Lehmann, B. and Jedwab, J. (2019) Orthocuproplatinum, IMA 2018-124. CNMNC Newsletter No. 47, February 2019, page 145; Mineralogical Magazine, 83, 143-147.

\section{IMA No. 2018-126}

Nickolayite

FeMoP

Daba-Siwaqa complex, Transjordan Plateau, Hatrurim Formation, Jordan ( $\left.31^{\circ} 21^{\prime} 52^{\prime} \mathrm{N}, 36^{\circ} 10^{\prime} 55^{\prime} \mathrm{E}\right)$

Mikhail N. Murashko, Yevgeny Vapnik, Yury S. Polekhovsky, Vladimir V. Shilovskikh, Anatoly N. Zaitsev, Oleg S. Vereshchagin and Sergey N. Britvin*

*E-mail: sbritvin@gmail.com

New structure type

Orthorhombic: Pnma; structure determined

$a=5.9519(5), b=3.7070(3), c=6.8465(6) \AA$

3.238(21), 2.298(100), 2.226(9), 2.181(89), 2.113(26), 1.927(14), $1.838(18), 1.388(13)$

Type material is deposited in the collections of the Fersman Mineralogical Museum, Russian Academy of Sciences, Leninskiy Prospekt 18-2, Moscow 119071, Russia, registration number 5290/1

How to cite: Murashko, M.N., Vapnik, Y., Polekhovsky, Y.P., Shilovskikh, V.V., Zaitsev, A.M., Vereshchagin, O.S. and Britvin, S.N. (2019) Nickolayite, IMA 2018-126. CNMNC Newsletter No. 47, February 2019, page 146; Mineralogical Magazine, 83, 143-147.

\section{IMA No. 2018-128}

Reaphookhillite

$\mathrm{MgZn}_{2}\left(\mathrm{PO}_{4}\right)_{2} \cdot 4 \mathrm{H}_{2} \mathrm{O}$

Reaphook Hill, Martins Well, Flinders Ranges, South Australia, Australia (3123'39”S, 139¹7'54”E)

Peter Elliott*

${ }^{\star}$ E-mail: peter.elliott@adelaide.edu.au

The Mg analogue of parahopeite

Triclinic: $P \overline{1}$; structure determined

$a=5.759(1), \quad b=7.534(1), c=5.279(1) \AA, \alpha=93.44(3), \beta=$ 91.27(3), $\gamma=91.30(3)^{\circ}$

7.577(100), 4.461(24), 3.771(14), 3.158(13), 2.982(32), 2.880(27), $2.775(14), 2.668(13)$

Type material is deposited in the mineralogical collection of the South Australian Museum, North Terrace, Adelaide, South Australia, Australia, registration number G34798

How to cite: Elliott, P. (2019) Reaphookhillite, IMA 2018-128. CNMNC Newsletter No. 47, February 2019, page 146; Mineralogical Magazine, 83, 143-147.

\section{IMA No. 2018-129}

Davidbrownite- $\left(\mathrm{NH}_{4}\right)$

$\left(\mathrm{NH}_{4}\right)_{5}\left(\mathrm{~V}^{4+} \mathrm{O}\right)_{2}\left(\mathrm{C}_{2} \mathrm{O}_{4}\right)\left[\mathrm{PO}_{2.75}(\mathrm{OH})_{1.25}\right]_{4} \cdot 3 \mathrm{H}_{2} \mathrm{O}$

Rowley mine (125-foot level), ca. $20 \mathrm{~km} \mathrm{NW}$ of Theba, Maricopa Co., Arizona, USA (332'57”N, 1131'59”W)

Anthony R. Kampf*, Mark A. Cooper, George R. Rossman, Barbara P. Nash, Frank C. Hawthorne and Joe Marty
*E-mail: akampf@nhm.org

New structure type

Monoclinic: $P 2_{1} / c$; structure determined

$a=10.356(6), b=8.923(5), c=13.486(7) \AA, \beta=92.618(9)^{\circ}$

10.39(100), 6.73(50), 5.97(34), 3.789(35), 3.160(47), 3.104(42),

3.043(35), 2.977(44)

Cotype material is deposited in the mineralogical collections of the Natural History Museum of Los Angeles County, 900 Exposition Boulevard, Los Angeles, CA 90007, USA, catalogue numbers 66951, 66955 and 66959

How to cite: Kampf, A.R., Cooper, M.A., Rossman, G.R., Nash, B.P., Hawthorne, F.C. and Marty, J. (2019) Davidbrownite, IMA 2018-129. CNMNC Newsletter No. 47, February 2019, page 146; Mineralogical Magazine, 83, 143-147.

\section{IMA No. 2018-006a}

Ognitite

NiBiTe

Ognit (or Medek) dunite-wehrlite complex, southern margin of the Siberian Craton, Irkutskaya oblast, Eastern Sayans, Russia Andrei Y. Barkov*, Luca Bindi, Björn Winkler, Wolfgang Morgenroth, Gennadiy I. Shvedov, Robert F. Martin, Federica Zaccarini, Camelia V. Stan, Nobumichi Tamura and Christopher J. Stanley

*E-mail: ore-minerals@mail.ru

Structurally related to melonite

Trigonal: $P 3 \mathrm{~m} 1$; structure determined

$a=3.9332(4), c=5.3920(6) \AA$

5.39(10), 3.39(15), 2.880(100), 2.697(10), 2.110(30), 1.968(30), 1.625(15), $1.589(20)$

Type material is deposited in the mineralogical collections of the Museo di Storia Naturale, Sezione di Mineralogia e Litologia, Università di Firenze, Via La Pira 4, I-50121, Firenze, Italy, catalogue number 3292/I

How to cite: Barkov, A.Y., Bindi, L., Winkler, B., Morgenroth, W., Shvedov, G.I., Martin, R.F., Zaccarini, F., Stan, C.V., Tamura, N. and Stanley, C.J. (2019) Ognitite, IMA 2018-006a. CNMNC Newsletter No. 47, February 2019, page 146; Mineralogical Magazine, 83, 143-147.

IMA No. 2018-051a

$\mathrm{CaZn}_{2}\left(\mathrm{AsO}_{4}\right)_{2} \cdot 4 \mathrm{H}_{2} \mathrm{O}$

Plaka Mine No. 80, Lavrion District Mines, Attikí Prefecture, Greece (374' $\left.18^{\prime \prime} \mathrm{N}, 24^{\circ} 1^{\prime} 54^{\prime} \mathrm{E}\right)$

Branko Rieck*, Gerald Giester, Christian Lengauer and Dan Topa

*E-mail: rieckb49@univie.ac.at

Structurally related to phosphophyllite

Monoclinic: $P c$; structure determined

$a=9.4160(3), b=5.3000(2), c=10.8931(4) \AA, \beta=91.767(1)^{\circ}$

9.406(100), 4.619(80), 3.612(35), 3.494(35), 2.984(60), 2.922(50), $2.720(20), 2.647(25)$

Type material is deposited in the mineralogical collections of the Natural History Museum-Vienna, Burgring 7, 1010 Vienna, Austria, inventory number O342

How to cite: Rieck, B., Giester, G., Lengauer, C. and Topa, D. (2019) IMA 2018-051a. CNMNC Newsletter No. 47, February 2019, page 146; Mineralogical Magazine, 83, 143-147. 
IMA No. 2018-055a

Bouškaite

$\left(\mathrm{MoO}_{2}\right)_{2} \mathrm{O}\left(\mathrm{SO}_{3} \mathrm{OH}\right)_{2}\left(\mathrm{H}_{2} \mathrm{O}\right)_{4}$

On the mine dump of the shaft Lill (total depth $454.4 \mathrm{~m}$ ), close to Příbram, central Bohemia, Czech Republic ( $49^{\circ} 41^{\prime} 49^{\prime} \mathrm{N}, 14^{\circ}$ 0'1"E)

Jiri Sejkora*, Ian E. Grey, Anthony R. Kampf, Jakub Plášil and Pavel Škácha

${ }^{\star}$ E-mail: jiri_sejkora@nm.cz

New structure type

Triclinic: $P \overline{1}$; structure determined

$a=5.581(3), b=9.572(1), c=14.425(4) \AA, \alpha=97.43(1), \beta=$ $100.05(2), \gamma=89.96(1)^{\circ}$

14.15(35), 7.078(100), 5.483(6), 5.440(9), 4.838(7), 4.720(56), 4.010(7), 3.240(10)

Type material is deposited in the collections of the Department of Mineralogy and Petrology, National Museum Prague, Cirkusová 1740, CZ-193 00 Praha 9, Czech Republic, catalogue number P1P 24/2018 (holotype), and the Natural History Museum of Los Angeles County, 900 Exposition Boulevard, Los Angeles, CA 90007, USA, catalogue number 66776 (cotype)

How to cite: Sejkora, J., Grey, I.E., Kampf, A.R., Plášil, J. and Škácha, P. (2019) Bouškaite, IMA 2018-055a. CNMNC Newsletter No. 47, February 2019, page 147; Mineralogical Magazine, 83, 143-147.

\section{IMA No. 2018-063a}

\section{Kenngottite}

$$
\mathrm{Mn}_{3}^{2+} \mathrm{Fe}_{4}^{3+}\left(\mathrm{PO}_{4}\right)_{4}(\mathrm{OH})_{6}\left(\mathrm{H}_{2} \mathrm{O}\right)_{2}
$$

Stannum mine, 5 th level of the Huber shaft, Krásno ore district, near Horní Slavkov, western Bohemia, Czech Republic (5007'22”N, 1248'2"E)

Jiří Sejkora*, Ian E. Grey and Anthony R. Kampf

${ }^{\star}$ E-mail: jiri_sejkora@nm.cz

Structurally related to souzalite and gormanite

Monoclinic: $P 2 / a$; structure determined

$a=13.909(10), b=5.186(4), c=12.159(9) \AA ̊ \Omega, \beta=98.88(1)^{\circ}$

4.87(47), 3.458(89), 3.209(100), 3.023(31), 2.623(46), 2.429(49), $1.951(28), 1.577(34)$
Type material is deposited in the collections of the Department of Mineralogy and Petrology, National Museum Prague, Cirkusová 1740, CZ-193 00 Praha 9, Czech Republic, catalogue number P1P 26/2018 (holotype), and the Natural History Museum of Los Angeles County, 900 Exposition Boulevard, Los Angeles, CA 90007, USA, catalogue number 66795 (cotype)

How to cite: Sejkora, J., Grey, J.E. and Kampf, A.R. (2019) Kenngottite, IMA 2018-063a. CNMNC Newsletter No. 47, February 2019, page 147; Mineralogical Magazine, 83, 143-147.

\section{IMA No. 2018-086a}

Edscottite

$\mathrm{Fe}_{5} \mathrm{C}_{2}$

Wedderburn meteorite, found $4.5 \mathrm{~km} \mathrm{NE}$ of Wedderburn, Victoria, Australia ( $\left.36^{\circ} 26^{\prime} \mathrm{S}, 143^{\circ} 38^{\prime} \mathrm{E}\right)$

Chi Ma and Alan Rubin

*E-mail: chi@gps.caltech.edu

Known synthetic analogue

Monoclinic: $\mathrm{C} 2 / \mathrm{c}$

$a=11.57, b=4.57, c=5.06 \AA, \beta=97.7^{\circ}$

$2.207(31), \quad 2.192(26), \quad 2.079(68), \quad 2.050(100), \quad 2.027(27)$, 2.010(36), 1.921(26), 1.816(22)

Type material is deposited in the meteorite collections of the Department of Earth, Planetary and Space Sciences, University of California, Los Angeles, California 90095-1567, USA, polished thick section ULCA 143

How to cite: Ma, C. and Rubin, A. (2019) Edscottite, IMA 2018-086a. CNMNC Newsletter No. 47, February 2019, page 147; Mineralogical Magazine, 83, 143-147.

\section{REVISED CHEMICAL FORMULAE}

A paper on the mineral satimolite has been recently published [Mineralogical Magazine, 82 (2018), 1033-1047] in which the ideal chemical formula of the mineral is given as $\mathrm{KNa}_{2}\left(\mathrm{Al}_{5} \mathrm{Mg}_{2}\right)$ $\left[\mathrm{B}_{12} \mathrm{O}_{18}(\mathrm{OH})_{12}\right](\mathrm{OH})_{6} \mathrm{Cl}_{4} \cdot 4 \mathrm{H}_{2} \mathrm{O}$, with magnesium as an essential constituent. These data were examined carefully by the CNMNC officers and were considered reliable. Accordingly it was agreed to modify the formula of satimolite in the official IMA List of Minerals. 